\title{
Expression of the Human Histocompatibility Leukocyte Antigen DR3 Transgene Reduces the Severity of Demyelination in a Murine Model of Multiple Sclerosis
}

\author{
Kristen M. Drescher, ${ }^{\ddagger}$ Loc T. Nguyen, ${ }^{\star}$ Veena Taneja, ${ }^{\star}$ Michael J. Coenen, ${ }^{\star}$ Julian L. Leibowitz, ${ }^{\S}$ Gundrun Strauss, \\ Gunter J. Hammerling, Chella S. David, ${ }^{\star}$ and Moses Rodriguez ${ }^{\star \ddagger}$ \\ *Department of Immunology and ${ }^{\ddagger}$ Department of Neurology, Mayo Clinic, Rochester, Minnesota 55905; ${ }^{\S}$ Department of Pathology and \\ Laboratory Medicine, Texas A and M University, College Station, Texas 77843-1114; and ${ }^{\|}$Tumor Immunology Program, German Cancer \\ Research Center, 69120 Heidelberg, Germany
}

\begin{abstract}
The role of various MHC genes in determining the progression of multiple sclerosis (MS) remains controversial. The HLA-DR3 gene has been associated with benign relapsing MS in some genetic epidemiologic studies, but with disease progression in others. We induced demyelination in highly susceptible B10.M and B10.Q mice expressing the DR3 (HLA-DRB1*0301) transgene to determine directly the effects of a human transgene by infecting them with Theiler's murine encephalomyelitis virus (TMEV). DR3 + mice experienced a dramatic reduction in the extent and severity of demyelination compared with DR3 - littermate controls, whereas anti-TMEV antibody titers, delayed-type hypersensitivity responses, and levels of infectious virus, virus antigen, and virus RNA were similar in both groups. To address a possible mechanism of how the human transgene is reducing virus-induced demyelination, we analyzed cytokine expression in the lesions and also determined whether B10.M mice can respond to peptides derived from the DR3 molecule. Intense staining for IFN- $\gamma$ and IL-4, T helper (TH) 1 and TH2 cytokines, respectively, was found in the lesions of TMEV-infected DR3 - mice but not in the DR3 + transgenic mice at day 21 after infection. DR3 peptides elicited strong proliferative responses in B10.M mice but not in B10.M (DR3+) mice. These experiments are the first to demonstrate that a human class II DR gene can alter the severity of demyelination in an animal model of MS without influencing viral load. These experiments are consistent with a mechanism by which DR3 reduces demyelination by altering the cytokine expression in the lesions, possibly by deleting $\mathrm{T}$ cells involved in virus-induced pathology. $(J$. Clin. Invest. 1998. 101:1765-1774.) Key words: Theiler's virus $\bullet$ demyelination
\end{abstract}

Portions of this work have been published in abstract form (1996. Eur. J. Neurol. 3:35).

Address correspondence to Moses Rodriguez, Department of Immunology, Mayo Clinic, 200 First Street, S.W., Rochester, MN 55901. Phone: 507-284-4664; FAX: 507-284-1637; E-mail: rodriguez.moses@ mayo.edu

Received for publication 18 March 1997 and accepted in revised form 12 February 1998.

The Journal of Clinical Investigation

Volume 101, Number 8, April 1998, 1765-1774

http://www.jci.org

\section{Introduction}

Both autoimmune (scleroderma [1], rheumatoid arthritis [2-4], insulin-dependent diabetes mellitus [5], and Graves' disease [6]) and infectious (hantavirus [7], herpes simplex virus [8], and coxsackievirus [9]) diseases have been shown to be influenced by MHC alleles. In most cases, the MHC genes are not the sole determinant of disease, but these genes impact either the severity or incidence of disease.

Despite intensive study, the genetic factors influencing the severity of multiple sclerosis (MS), ${ }^{1}$ the most common primary demyelinating disease of the central nervous system (CNS), are not fully understood. Although genes of the MHC were first implicated in MS in 1972 (10), lack of complete penetrance of the MHC genes associated with susceptibility, combined with environmental factors, has led to controversy concerning the alleles critical for disease development, and in particular the genetic factors that distinguish between benign relapsing disease and chronic progressive disability. Several recent studies involving complete genome screens have been unable to reach a consensus as to the critical genes involved in the development of the disease (11-13). Many of the associations made to date are not absolute and vary according to ethnic group. For example, among individuals of Northern European origin, strong associations have been identified between particular MHC class I (HLA A3 and B7) and class II (DR2 and DQW1) alleles and the development of MS (reviewed by Ebers and Sadovnick [14]).

The DR3 gene has been associated with benign relapsing MS in some studies, whereas others have shown that DR3 is associated with a poor outcome. In a study by Duquette et al. (15), patients were defined as having severe or benign MS based on their Kurtzke score over a period of $10 \mathrm{yr}$ or more. Analysis of HLA antigen subtypes demonstrated an increased frequency of HLA-DR2 in both the benign and severe forms of the disease compared with control patients, but an association between DR3 and the absence of disease progression was found. Although the association between DR3 and benign MS has been observed in other populations (16), some investigators $(17,18)$ have found no correlation, or the opposite association, i.e., progressive disease with DR3 (19).

Intracerebral injection of susceptible mice with Theiler's murine encephalomyelitis virus (TMEV) results in an immune-mediated demyelinating disease with pathology similar to MS (20). Immunogenetic studies have demonstrated a role for both class I and class II MHC gene products as determi-

1. Abbreviations used in this paper: $\mathrm{DTH}$, delayed-type hypersensitivity; MS, multiple sclerosis; PFU, plaque-forming units; TH, T helper; TMEV, Theiler's murine encephalomyelitis virus. 
nants of resistance or susceptibility to TMEV-induced demyelination (21-26). The class I MHC D region determines primarily whether or not virus will be cleared from the CNS, or whether virus will persist and demyelination will ensue (21-23). However, several lines of evidence support an important role of class II genes in demyelination $(a)$ alleles of the H2-A and $\mathrm{H} 2$-E loci do not influence the incidence of demyelinating disease, but affect the severity of demyelination (26); (b) mAb therapy directed against H2-A $(27)$ or CD4 $(28,29)$ at the time of virus infection increases mortality, whereas treatment after demyelination is established (day 15 after infection) diminishes myelin injury (27); (c) class II knockout mice on a resistant $\mathrm{H}-2^{\mathrm{b}}$ haplotype develop high virus titers, clinical deficits, and small foci of demyelination, further supporting the role of class II-restricted CD4 T cells in protection from TMEV infection (30); and $(d)$ class II gene products are upregulated in CNS glial cells during persistent virus infection, which correlates with development of demyelination (31).

To address the controversy for the role of human HLADR3 gene in the progression of MS, we tested the role of a human gene in B10.M and B10.Q mice, which are extremely susceptible to TMEV-induced demyelination. The availability of a well-characterized murine model of virus-induced demyelination, coupled with the technology to create "humanized" mice with the DR3 transgene, provided us with the unique opportunity to examine the role of this particular HLA gene in disease progression. Mice expressing the human DR3 transgene had significantly decreased severity of demyelination compared with littermate controls, correlating with the human studies which found DR3 associated with benign relapsing MS (15) rather than progressive disability. The mechanism for the reduction in demyelination appears to be the result of altering the profile of cytokine expression early in the white matter lesion.

\section{Methods}

Virus. The Daniel's strain of TMEV was used in all experiments. The history of this virus has been described previously (20).

DR3 (Drw17) transgenic mice. DR3 (Drw17) transgenic mice were generated by coinjection of the HLA-DRA genomic fragment and a DRB1*0301B gene fragment into embryos from $(\mathrm{C} 57 \mathrm{Bl} / 6 \times$ DBA/2)F1 donors, mated to C57B1/10 males as described previously (32). These mice were subsequently backcrossed to B10.M and B10.Q mice at the Mayo Clinic Immunogenetics Mouse Facility (Rochester, $\mathrm{MN}$ ) for 10 generations, and were therefore considered congenic. Nontransgenic littermate B10.M and B10.Q controls were used for all comparisons. Mice were screened by flow cytometry to detect DR3 expression in blood lymphocytes as described previously (32). Previously published studies have demonstrated that high levels of transgene RNA are expressed in the spleen and thymus, intermediate levels in the lung and kidney, and low or no expression in the liver, heart, and brain. The level of expression of the DR3 protein was similar to that of the endogenous mouse class II molecules (32). All mice in these studies were treated in accordance with the National Institutes of Health Guidelines for the Care and Use of Animals in Research.

Infection, harvesting, and morphology of the CNS. At 4-6 wk of age, mice were infected intracerebrally with $2 \times 10^{5}$ plaque-forming units (PFU) of TMEV in a total volume of $10 \mu l$. At day 45 after infection, mice were perfused with Trump's fixative by intracardiac puncture. Spinal cord blocks $(1 \mathrm{~mm})$ were embedded in glycol methacrylate and stained with a modified erichrome stain with cresyl violet counterstain (33). Morphologic analysis was performed on 10-15 coronal spinal cord sections from each animal. A total of 1,490 spinal cord quadrants were studied. A pathologic score was determined for each animal based on meningeal inflammation, demyelination, and neuronal inflammation. Grading was performed on coded sections in a blinded manner without knowledge of genotype. Each quadrant from every third coronal section from each mouse was scored for the presence of pathological abnormalities. The score was expressed as a percent of the total number of quadrants examined per mouse. A maximum pathologic score of 100 indicated that there was disease in all quadrants of every spinal cord block from a particular mouse. In addition, some mice were killed by overdose of pentobarbital at 7, 21, 45, and $90 \mathrm{~d}$ after infection, and the tissues were frozen in OCT embedding compound (Miles, Inc., Elkhart, IN) for immunostaining and in situ hybridization. White matter and lesion area were measured using an interactive image analysis system attached to a photomicroscope (Carl Zeiss, Inc., Thornwood, NY).

Virus plaque assay. At days 7 and 45 after infection, the brains and spinal cords from transgenic and nontransgenic mice were removed after killing, and a $10 \%$ (wt/vol) homogenate was prepared in DME (GIBCO BRL, Gaithersburg, MD). The homogenate was clarified by centrifugation and stored at $-70^{\circ} \mathrm{C}$ until the time of assay. The assay was performed on L2 cells as described previously (34), in duplicate.

Immunoperoxidase staining. $10-\mu \mathrm{m}$-thick sections were cut and fixed in chilled acetone. Sections were stained with $\mathrm{mAbs}$ to either CD4+ or CD8+ T cells (PharMingen, San Diego, CA), HLA-DR3 (PharMingen), IFN- $\gamma$ (PharMingen), TNF- $\alpha$ (PharMingen), IL-4 (PharMingen), or a polyclonal rabbit anti-TMEV sera, using the avidin-biotin immunoperoxidase technique (Vector Laboratories, Inc., Burlingame, CA) as described previously (35). Slides were developed with Hanker Yates reagent (Polysciences Inc., Warrington, PA), using hydrogen peroxide as the substrate. Slides were lightly counterstained with Mayer's hematoxylin.

In situ hybridization. Nick translation was used to obtain a probe with a specific activity between 5 and $8 \times 10^{7} \mathrm{cpm}$ of $\left[\alpha{ }^{35} \mathrm{~S}\right] \mathrm{dATP} / \mu \mathrm{g}$ of DNA. Frozen sections were hybridized with a $253-b p{ }^{35} \mathrm{~S}$-labeled probe specific for the VP-1 region of TMEV and exposed for $48 \mathrm{~h}$ as described previously (36). The CNS area was measured with an image analysis system (IBAS; Kontron Electronik, Eching, Germany) attached to an Axiophot microscope (Carl Zeiss, Inc.), to determine the number of virus RNA-positive cells per spinal cord area $\left(\mathrm{mm}^{2}\right.$ [36]).

Anti-TMEV IgG ELISA. Blood was obtained from mice at the time of killing and allowed to clot, and the sera were stored at $-80^{\circ} \mathrm{C}$ until the time of assay. Polystyrene microtiter plates (Immunolon II; Dynatech Laboratories, Inc., Chantilly, VA) were coated with $0.5 \mu \mathrm{g}$ of purified TMEV in $0.1 \mathrm{M}$ carbonate buffer ( $\mathrm{pH}$ 9.5), then blocked with $1 \%$ BSA (Sigma Chemical Co., St. Louis, MO) in PBS. Sera from individual mice were diluted in $0.2 \%$ BSA and incubated at room temperature. Biotinylated goat anti-mouse IgG (Jackson ImmunoResearch Labs, West Grove, PA) was used as the secondary antibody. Detection was performed using alkaline phosphatase-labeled streptavidin (Jackson ImmunoResearch Labs), with para-nitrophenylphosphate used as the substrate. Absorbances were read at $\mathrm{A}_{405}$ (30).

Delayed-type hypersensitivity (DTH) responses to TMEV. TMEVspecific DTH responses were assessed as described previously (30). Briefly, $2.5 \mu \mathrm{g}$ of ultraviolet-irradiated purified TMEV was injected intradermally in the ear, and ear swelling was measured at 24 and $48 \mathrm{~h}$ after injection with a dial gauge micrometer and compared with the preinjection measurement.

In vitro proliferation assay. The peptides tested in this experiment corresponded to the HV3 region of $\mathrm{E}_{\beta}^{\mathrm{d}}$ (65-79), DR2Dw21, and Dw3. All peptides were synthesized by previously described procedures (37). B10.M mice were injected with $200 \mu \mathrm{g}$ of peptides emulsified in CFA. In some experiments, B10.M (DR3+) mice were injected with the same concentration of Dw3 peptide. $7 \mathrm{~d}$ later, draining lymph nodes were removed, and a single cell suspension was prepared. Lymph node cells $\left(10^{6}\right.$ cells/well; $100 \mu \mathrm{l}$ vol $)$ were cocultured 
with $100 \mu \mathrm{l}$ of peptide at a final concentration of $100 \mu \mathrm{g} / \mathrm{ml}$ as described previously $(3) .\left[{ }^{3} \mathrm{H}\right]$ Thymidine $(1.2 \mu \mathrm{Ci})$ was added for the last $18 \mathrm{~h}$ of culture. Cells were harvested on glass fiber filters, and $\left[{ }^{3} \mathrm{H}\right]$ thymidine was determined in a liquid scintillation counter (LKB Wallac, Turku, Finland). Additional cells were cocultured for $72 \mathrm{~h}$ with Con A (10 $\mu \mathrm{g} / \mathrm{ml}$; Sigma Chemical Co.). All cultures were performed in triplicate. Results are expressed as a stimulation index calculated by dividing the mean counts per minute from the test well by the mean counts per minute from the medium control wells.

Statistics. Analysis was performed using either the Student's $t$ test if the data were normally distributed or the Mann-Whitney Rank Sum test if the data were not normally distributed.

\section{Results}

$H L A-D R 3$ reduces the extent of TMEV-induced demyelination in susceptible B10.M and B10.Q mice. To test whether expression of the human DR3 transgene altered the susceptibility of mice to TMEV-induced demyelination, we compared the extent of gray matter inflammation, meningeal inflammation, and demyelination in transgenic mice and nontransgenic littermate controls at day 45 after infection. This particular time after infection was chosen because it has been used previously to determine whether mice are resistant or susceptible to demyelination (25), and to assess therapeutic efficacy of immunomodulatory agents (28). Expression of HLA-DR3 reduced the severity of demyelination in the spinal cord in B10.M mice. There was an almost fourfold decrease in the demyelination scores of DR3 + transgenic $(4.1 \pm 1.3)$ versus DR3 - nontransgenic mice $(15.5 \pm 3.3 ; P<0.01$; Table I). The lesions in the DR3 + mice were significantly smaller than those of the transgene-negative controls $\left(0.07 \pm 0.02\right.$ vs. $0.57 \pm 0.12 \mathrm{~mm}^{2} ; P=$ $0.001)$. There was also a reduction in meningeal inflammation in the DR3 + mice, but this was not statistically significant (Table I). While the extent of demyelination was reduced markedly in mice expressing the DR3 transgene, the incidence of demyelination was comparable between the transgenic mice and their littermate controls $(72.7 \%$ [ 8 of 11 mice with disease] vs. $81.3 \%$ [ 13 of 16 mice with disease], respectively). The extent of demyelination as determined by the percentage of spinal cord quadrants with demyelination between the nontrans-

Table I. Human DR3 Transgene Reduces the Extent of Demyelination in the Spinal Cord after TMEV Infection

\begin{tabular}{lrrrr}
\hline & & \multicolumn{3}{c}{ Mean pathology score \pm SEM } \\
\cline { 3 - 5 } \multicolumn{1}{c}{ Group } & $n$ & $\begin{array}{c}\text { Gray matter } \\
\text { inflammation }\end{array}$ & $\begin{array}{r}\text { White matter } \\
\text { inflammation }\end{array}$ & Demyelination \\
\hline B10.M (DR3+) & 11 & $0.0 \pm 0.0$ & $2.9 \pm 0.9$ & $4.1 \pm 1.3$ \\
B10.M (DR3-) & 16 & $0.2 \pm 0.1$ & $12.1 \pm 3.0$ & $15.5 \pm 3.3$ \\
B10.M & 5 & $0.8 \pm 0.5$ & $17.3 \pm 5.0$ & $20.5 \pm 4.2$ \\
B10.Q (DR3+) & 11 & $0.4 \pm 0.3$ & $2.5 \pm 0.9$ & $6.7 \pm 2.0$ \\
B10.Q (DR3-) & 6 & $2.1 \pm 1.8$ & $17.1 \pm 3.8$ & $22.9 \pm 4.0$ \\
& & & & \\
\hline
\end{tabular}

For each mouse, 10-15 spinal cord sections were graded for gray matter inflammation, white matter inflammation, and demyelination. The data are expressed as the percentage of spinal cord quadrants with disease (mean \pm SEM). Differences between demyelination scores were statistically significant between B10.M (DR3+) and B10.M (DR3-) $(P=$ $0.01)$ and between B10.Q (DR3+) and B10.M (DR3-) mice $(P=0.02)$ by Student's $t$ test.
Table II. Virus Titers in the CNS of TMEV-infected Transgenic (DR3+) and Nontransgenic (DR3-) Mice

\begin{tabular}{crrr}
\hline Group & Day* & $n$ & Virus titer \\
\hline B10.M (DR3-) & 7 & 3 & $5.23 \pm 0.12$ \\
B10.M (DR3+) & 7 & 3 & $4.93 \pm 0.34$ \\
B10.M (DR3-) & 45 & 3 & $2.55 \pm 0.12$ \\
B10.M (DR3+) & 45 & 3 & $2.56 \pm 0.17$
\end{tabular}

Virus titers were determined by viral plaque assay on L2 cells. Results are expressed as the virus titer per gram of CNS tissue $\left(\log _{10} \mathrm{PFU}\right)$ mean \pm SEM. The detection limit of the assay was $1.7 \log _{10} \mathrm{PFU} / \mathrm{g}$ of CNS tissue. Differences between the two groups were not found to be statistically significant by the Student's $t$ test. *Day after infection with TMEV.

genic littermate controls and the parental B10.M mice was comparable ( 15.5 vs. $20.5 \%$ ). The lesions observed in both the DR3 transgenic and nontransgenic mice were localized almost exclusively to the white matter, with areas containing multiple demyelinated axons (Fig. 1, $A$ and $B$ ).

To address whether the protective effect of DR3 was generalized to other susceptible mouse $\mathrm{H}-2$ haplotypes, we generated DR3 human transgenics on susceptible B10.Q (H-2q) haplotypes. Similar reductions in demyelination were observed in B10.Q DR3+ mice compared with transgene-negative littermate controls (Table $\mathrm{I} ; P=0.02$ ). This provided support for the conclusion that the mechanism by which DR3 reduces the severity of demyelination is independent of mouse $\mathrm{H}-2$ haplotype.

HLA-DR3 transgene expression does not effect virus levels in the CNS. To test whether the mechanism of reduced demyelination in the DR3 transgenic mice is related to a decrease in virus burden, virus plaque assays were performed at days 7 and 45 after infection and compared with results obtained with tissues from infected littermate nontransgenic mice. As previous studies from our laboratory have determined that day 7 is when peak virus titers are detected (35), day 7 was one time point chosen for study. Virus titers at day 7 after infection were similar in transgenic and nontransgenic mice such that $4-5 \log _{10}$ $\mathrm{PFU} / \mathrm{g}$ of CNS tissue were detected (Table II). Likewise, at day 45 after infection, a time point considered to be the chronic phase of infection, infectious titers were equivalent, such that $2-3 \log _{10}$ of infectious virus were detected. This indicated that the mechanism by which the DR3 transgene ameliorates demyelination is not by preventing virus persistence. This result was of particular importance because previous data in the model have indicated a close correlation between virus persistence and demyelination (30).

To address whether the DR3 transgene may have had a local effect of decreasing virus antigen or RNA expression in the lesion, we performed immunostaining with a polyclonal antibody to TMEV and in situ hybridization with a VP-1-specific probe on the spinal cords of transgenic and nontransgenic mice. Similar numbers of cells positive for virus RNA per square millimeter of spinal cord were detected by in situ hybridization in both the transgenic and nontransgenic mice at days 7, 21, 45, and 90 after infection (Table III). Immunostaining of spinal cord sections with an antibody to TMEV demonstrated that virus antigen levels were similar between the two 


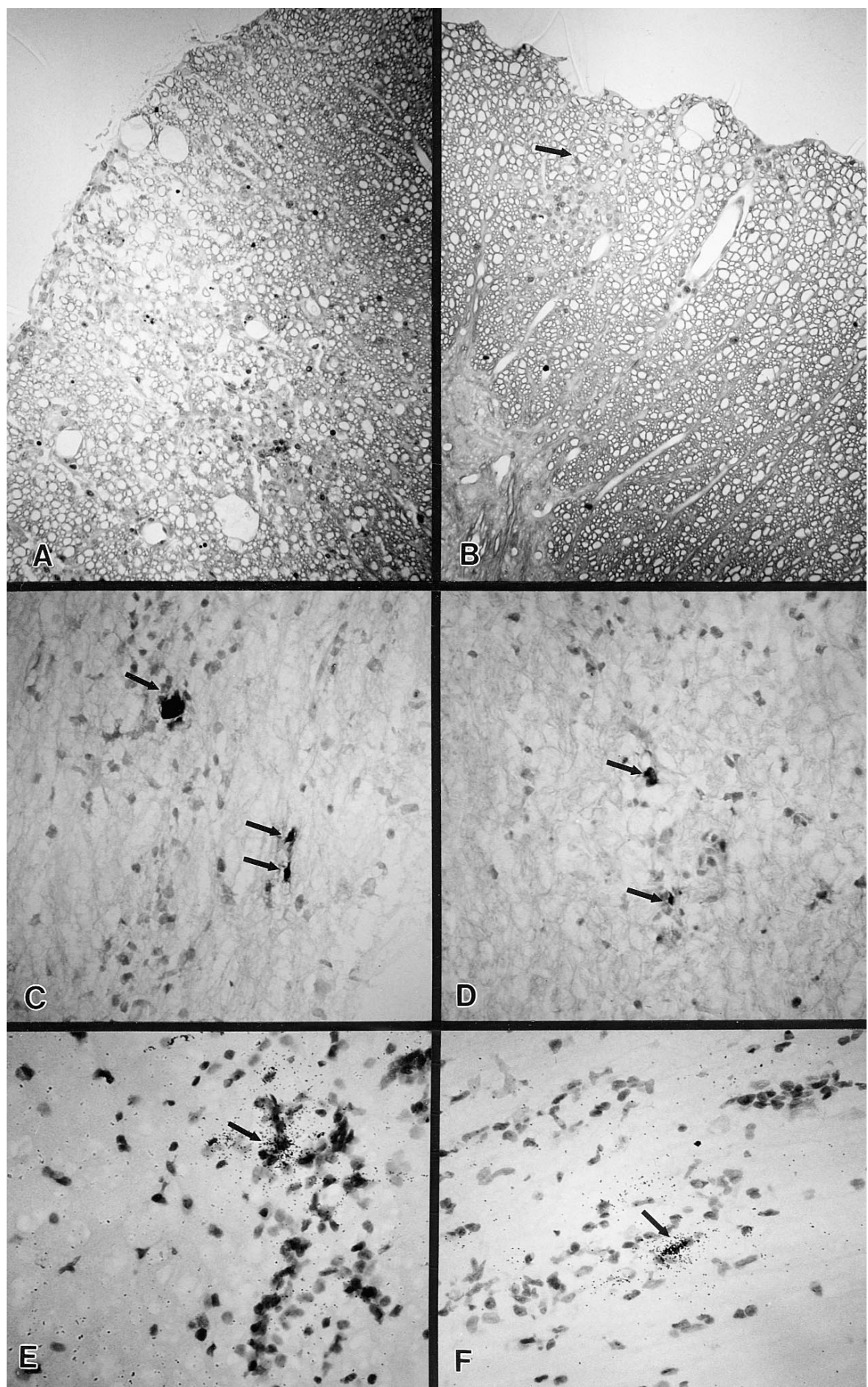

Figure 1. Demyelination in the spinal cord of B10.M (DR3-) nontransgenic $(A)$ compared with B10.M (DR3+) transgenic mice $(B)$ at day 45 after infection with TMEV. Note increased number of demyelinated axons in the spinal cord of DR3- compared with DR3 + mice. On average, the size and the number of lesions were less in DR3 + versus DR3 - mice. Spinal cord sections were embedded in glycol methacrylate plastic and stained with a modified erichrome/cresyl violet stain. Localization of TMEV antigen ( $C$ and $D$ ) and RNA $(E$ and $F$ ) in the spinal cords of B10.M (DR3 -) nontransgenic ( $D$ and $F$ ) and B10.M (DR3+) transgenic ( $C$ and $E$ ) mice at day 21 after infection. Virus antigenpositive cells ( $C$ and $D$ ) are identified by the dark immunoperoxidase reaction products (arrows). In situ hybridization was performed with a ${ }^{35} \mathrm{~S}$-labeled probe specific for the VP-1 region of TMEV. Virus RNApositive cells $(E$ and $F$ ) are identified by silver grains (arrows). Frozen sections $(C, D, E$, and $F$ ) were lightly counterstained with Mayer's hematoxylin. groups (Fig. 1, $E$ and $F$ ). At day 45 after infection, $0.52 \pm 0.18$ virus antigen-positive cells $/ \mathrm{mm}^{2}$ examined were detected in the spinal cords of DR3 - mice $(n=13)$, whereas in DR3+ mice $(n=11), 0.23 \pm 0.08$ virus antigen-positive cells $/ \mathrm{mm}^{2}$ were detected. Therefore, the mechanism by which DR3 was decreasing demyelination did not involve limiting local virus replication.

Expression of HLA-DR3 transgene does not alter the proportion of $C D 4+$ versus $C D 8+T$ cells in the demyelinating lesion. Previous work in the TMEV model has shown that by depleting either the CD4+ or CD8+ T cells from the lesion, it is possible to ameliorate virus-induced demyelination $(27,28)$. Therefore, it was important to test whether the mechanism of decreasing demyelination by the DR3 transgene was by altering the phenotype of T cells in the lesions. Previous work from our laboratory demonstrated that early in TMEV infection (7 d after infection), CD4+ T cells are the predominant $\mathrm{T}$ cell type in the CNS of susceptible mice (37). In the current study, no differences were found in the mean numbers of CD4+ or CD8 + T cells at any time point studied (days 7, 21, or 45 after 
Table III. Virus RNA in the Spinal Cords of TMEV-infected Transgenic (DR3+) and Nontransgenic (DR3-) Mice

\begin{tabular}{cccc}
\hline Group & Day after infection & $n$ & Positive cells $/ \mathrm{mm}^{2}$ \\
\hline B10.M (DR3+) & 7 & 3 & $0.12 \pm 0.04$ \\
B10.M (DR3-) & 7 & 3 & $0.26 \pm 0.10$ \\
B10.M (DR3+) & 21 & 4 & $0.20 \pm 0.06$ \\
B10.M (DR3-) & 21 & 4 & $0.09 \pm 0.07$ \\
B10.M (DR3+) & 45 & 4 & $0.42 \pm 0.30$ \\
B10.M (DR3-) & 45 & 2 & $0.34 \pm 0.20$ \\
B10.M (DR3+) & 90 & 3 & $0.16 \pm 0.04$ \\
B10.M (DR3-) & 90 & 2 & $0.41 \pm 0.36$ \\
& & & \\
\hline
\end{tabular}

Number of TMEV RNA-positive cells $/ \mathrm{mm}^{2}$ of spinal cord tissue as detected by in situ hybridization of frozen sections with a ${ }^{35} \mathrm{~S}$-labeled probe specific for the VP-1 region of TMEV. Differences between the two groups at each time point were not found to be statistically significant by the Student's $t$ test. Data expressed as mean \pm SEM.

infection). Three animals from each group were studied per time point. The $\mathrm{T}$ cell infiltrate at $7 \mathrm{~d}$ after infection in both the transgenic and nontransgenic mice consisted almost exclusively of CD4+ T cells, with only a few CD8+ T cells. By day 21 after infection, the mean number of CD4+ $\mathrm{T}$ cells per square millimeter of lesion was similar between the transgenic mice and littermate controls $(16.3 \pm 3.3$ and $13.4 \pm 3.8$, respectively), as was the number of $\mathrm{CD} 8+\mathrm{T}$ cells $(1.4 \pm 1.0$ and $5.4 \pm 2.8$ ). By day 45 after infection, the ratios of CD4+ to $\mathrm{CD} 8+\mathrm{T}$ cells were similar between the two groups of mice and approached the ratio of 3:1 reported previously by our laboratory (2.2:1 in the transgenic mice and 2.8:1 in littermate controls [38]).

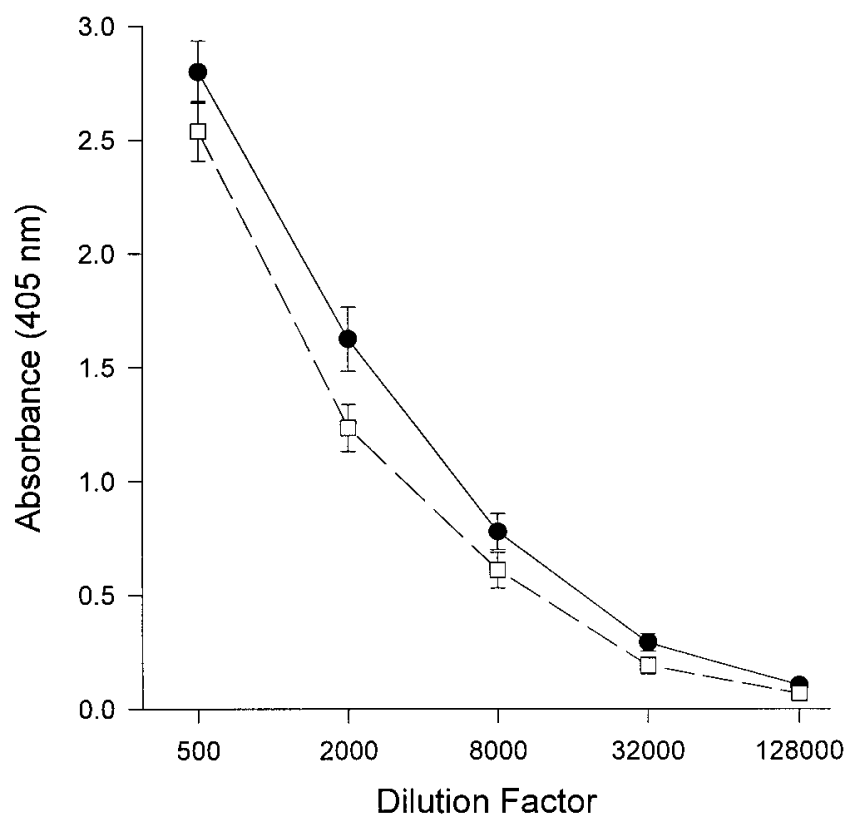

Figure 2. TMEV-specific IgG responses in B10.M (DR3+) transgenic and nontransgenic B10.M littermate control mice infected with TMEV for $45 \mathrm{~d}$. The data are expressed as the mean \pm SEM. No differences were observed between the groups. Circles, DR3- mice. Squares, DR3+ mice.
HLA-DR3 transgenic mice develop normal humoral immune responses and DTH responses to TMEV. Studies from our laboratory have demonstrated that immunoglobulins influence the level of demyelination resulting from TMEV infection in susceptible mouse strains (39). To determine the effect of HLA-DR3 on antibody production, TMEV-specific IgG was measured by ELISA to purified virus antigen at day 45 after infection. Both transgenic and nontransgenic mice developed similar antibody titers to TMEV (Fig. 2) compared with serum from uninfected mice.

Previous studies have shown that DTH responses to TMEV antigens correlate with demyelination (22). To determine if this relationship held true in this system, we measured the DTH response to purified TMEV antigens 3 mo after infection. At 24 and $48 \mathrm{~h}$ after intradermal injection, similar responses were obtained for both the transgenic mice and nontransgenic controls (Table IV). The antibody and DTH responses argue that the mechanism for decreasing demyelination by the DR3 transgene is not by interfering with T celldependent class II-mediated responses to viral antigens or peptides.

B10.M mice have DR3-responsive T cells. A potential mechanism of altering disease in DR3 transgenic mice involves the deletion of $\mathrm{T}$ cells involved in demyelination. To test if susceptible B10.M mice respond to peptides derived from the DR3 molecule, B10.M mice were immunized with DR3-derived peptides. The Dw2 peptide (derived from the DR2 molecule) was used as a negative control. A peptide derived from the endogenous mouse class II $\mathrm{E}_{\beta}$ gene was used as a positive control. In vitro proliferative responses were assessed using $\left[{ }^{3} \mathrm{H}\right]$ thymidine incorporation. The DR3 peptide elicited strong proliferative responses in B10.M mice (Table V), whereas mice immunized with the Dw2 peptide had minimal responses. The minimal response to the Dw2 peptide suggests that B10.M mice are tolerized to this peptide. A strong proliferative response was observed with the $\mathrm{E}_{\beta}$ peptide in $\mathrm{B} 6$ mice. This provided a potential mechanism by which recognition of DR3 as self may have resulted in deletion of $\mathrm{T}$ cells involved in pathogenesis.

B10.M (DR3+) mice do not have T cells that respond to the Dw3 peptide. To further demonstrate that Dw3-responsive $\mathrm{T}$ cells are deleted in the B10.M (DR3+) mice, B10.M (DR3+) mice were immunized with Dw3 peptide (derived from the DR3 molecule), and in vitro proliferative responses were assessed using $\left[{ }^{3} \mathrm{H}\right]$ thymidine incorporation. Despite a strong

Table IV. Effect of the Human DR3 Transgene on TMEV-specific DTH Responses

\begin{tabular}{cccc}
\hline & & \multicolumn{2}{c}{ Mean ear swelling $\left(10^{-2} \mathrm{~mm} \pm \mathrm{SEM}\right)$} \\
\cline { 3 - 4 } Group & $n$ & $24 \mathrm{~h}$ & $48 \mathrm{~h}$ \\
\hline B10.M (DR3+) & 6 & $5.83 \pm 1.01$ & $6.83 \pm 0.87$ \\
B10.M (DR3-) & 4 & $6.75 \pm 0.25$ & $7.25 \pm 0.48$
\end{tabular}

DTH responses were assayed on mice at day 90 after infection with TMEV. Purified TMEV antigens were injected intradermally into the ear, and swelling was assessed against preinjection measurements. Differences in mean ear swelling between the two groups at 24- and 48-h time points were not found to be statistically significant by the Student's $t$ test. 
Table V. Effect of the Human DR3 Transgene on In Vitro T Cell Proliferative Responses

\begin{tabular}{llcrr}
\hline Peptide & \multicolumn{1}{c}{ Strain } & Medium & Con A & Peptide \\
\hline E $^{\mathrm{d}}$ & B6 & $6579 \pm 73$ & $325000 \pm 10109$ & $53512 \pm 1903$ \\
Dw2 & B10.M & $6491 \pm 108$ & $401360 \pm 22449$ & $12562 \pm 111$ \\
Dw3 & B10.M & $11262 \pm 234$ & $334920 \pm 11307$ & 6073 \\
Dw3 & B10.M (DR3+) & $11773 \pm 735$ & $53665 \pm 2474$ & $111080 \pm 4421$ \\
& & & & $13025 \pm 5416$ \\
\end{tabular}

Mice were immunized with $200 \mu \mathrm{g}$ of peptide emulsified in CFA. Draining lymph nodes were removed $7 \mathrm{~d}$ later and cocultured with $100 \mu \mathrm{l}$ of peptide (final concentration of $100 \mu \mathrm{g} / \mathrm{ml})$, Con A $(10 \mu \mathrm{g} / \mathrm{ml})$, or medium alone. All cultures were performed in triplicate. Results are expressed as counts per minute. $\Delta \mathrm{cpm}$ is the difference in $\left[{ }^{3} \mathrm{H}\right]$ thymidine counts between peptide and the medium.

nonspecific proliferative response to Con A, the B10.M (DR3+) mice failed to mount an in vitro proliferative response to the DR3 peptide (Table V). This result demonstrates that despite a genetic predisposition of B10.M mice to mount a Dw3-specific response, the presence of the HLA-DR3 transgene abrogates this response.

Cytokine profiles in lesions of HLA-DR3 transgenic versus nontransgenic mice differ. An attractive mechanism by which the HLA-DR3 transgene reduces white matter pathology is that the transgene alters the expression of pathogenic cytokines early in the evolution of the demyelinating plaques. To determine whether the HLA-DR3 transgene effected the pattern of cytokines localized in the TMEV-induced lesions, immunostaining was performed using antibodies specific to murine TNF- $\alpha$, IFN- $\gamma$, and IL-4. These cytokines were studied because they are critical in the evaluation of T helper (TH) 1 (IFN- $\gamma, \mathrm{TNF}-\alpha$ ) versus TH2 (IL-4) immune responses. Three animals were examined at each time point. At day 7 after infection, minimal staining for both IFN- $\gamma$ and IL-4 was apparent in transgene-positive and -negative mice. By day 21 after infection, a time point when the demyelinating lesions are emerging, unique patterns of IFN- $\gamma$ and IL-4 staining were observed between the DR3+ mice and their littermate controls. Intense staining for both IFN- $\gamma$ and IL-4 was seen in the littermate controls (Fig. 3, $A$ and $C$ ) but not the transgene-positive mice (Fig. 3, $B$ and $D$ ). By day 45 after infection, IFN- $\gamma$ and IL-4 were observed in the lesions of both groups of mice (data not shown). Positive staining for TNF- $\alpha$ was obtained in both transgene-positive and -negative mice at all time points studied (days 7, 21, 45, and 90 after infection; Fig. 3, $E$ and $F$ ). No immunoreactivity for IFN- $\gamma$, IL-4, or TNF- $\alpha$ was observed in the CNS of uninfected transgene-positive or -negative mice (data not shown). These results demonstrate that the mechanism by which HLA-DR3 alters demyelination may involve the modulation of soluble mediator expression in the lesion early in the development of demyelinating disease. This change in cytokine expression may be directly related to the decreased numbers of inflammatory cells in the lesions of mice expressing HLA-DR3.

HLA-DR3 protein is expressed by CNS resident and nonresident cells in infected transgenic mice. Under normal conditions, mice do not express class II in the CNS. Previous work from our laboratory has demonstrated that after infection with TMEV, class II expression is induced on CNS resident cell types. We tested whether the effect of DR3 on white matter pathology is based on the expression of the gene on $\mathrm{T}$ cells or resident CNS cells. Immunostaining with an antibody specific for human MHC class II protein revealed that the lymphocytes infiltrating the lesions of the transgenic mice expressed high levels of DR3 (Fig. $4 A$ ). As expected, no positive staining for the transgene was detected in infected nontransgenic littermate controls (Fig. $4 \mathrm{~B}$ ), demonstrating that this antibody does not cross-react with any endogenous proteins of the mouse. Of particular interest, positive-staining cells with morphology reflective of neurons and astrocytes demonstrate that CNS resident cells are also induced to express human class II proteins after TMEV infection (Fig. $4 C$ ). No positive staining was observed in uninfected transgene-positive mice (Fig. $4 D$ ). These results suggest that the DR3 transgene could function by acting as an antigen-presenting molecule after the immune response to TMEV has been initiated, indicating that the transgene could directly modulate CNS pathology.

\section{Discussion}

Most of the focus in the study of the relationship between MS and $\mathrm{MHC}$ has been to determine the association of specific alleles with the frequency of disease at the population level. An association with the development of MS is apparent with both class I (A3 and B7) and class II (DR2 and DQW1) genes depending on the ethnicity of the groups studied (reviewed by Ebers and Sadovnick [14]). The role of various class I or class II genes in the progression of MS has been studied, but no consensus has been reached as to whether particular alleles are correlated with long-term disability. Because there has been controversy regarding the relationship between DR3 and benign relapsing MS, we used a well-characterized model of virus-induced demyelination to study the effects of the human DR3 gene in mice highly susceptible to TMEV infection. The severity but not the incidence of demyelination was reduced in B10.M and B10.Q mice expressing the human transgene compared with nontransgenic controls. Reduction of demyelination occurred without a corresponding decrease in viral replication or viral antigen expression. These results are the first to directly support human epidemiological studies that have associated the DR3 gene with benign relapsing MS.

The most obvious mechanism of reduced demyelination in the transgene-positive mice involves the DR3 molecule decreasing TMEV levels either directly or indirectly in the CNS. This mechanism was tested using three different measuresinfectious virus, TMEV RNA, and TMEV antigen. Using these three methods, no evidence of decreased viral burden was found, leading to the conclusion that the DR3 transgene 


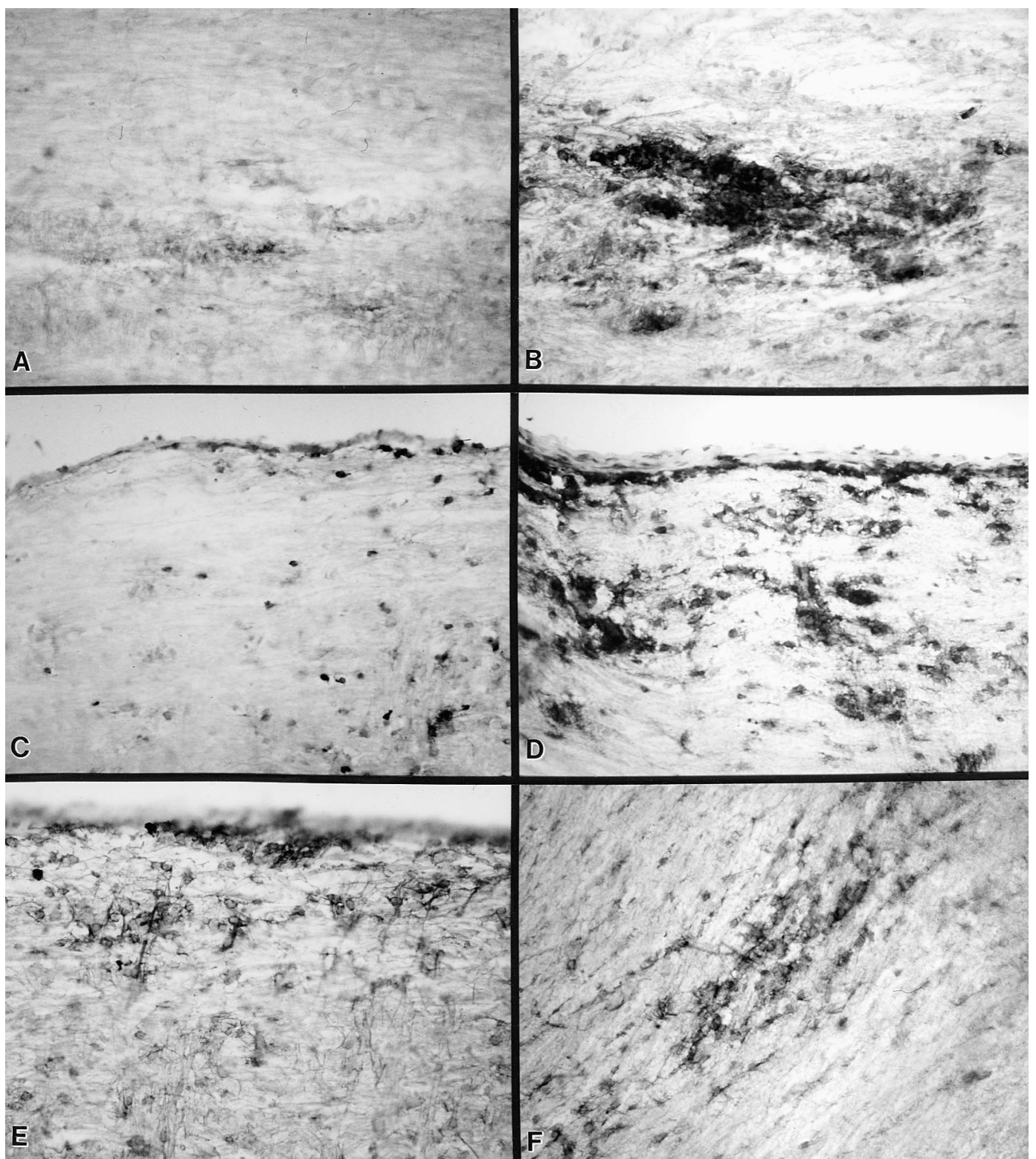

Figure 3. Cytokine immunoreactivity in the spinal cords of B10.M (DR3-) nontransgenic mice $(A, C$, and $E)$ and B10.M (DR3+) transgenic mice $(B, D$, and $F)$ at day 21 after infection with TMEV. Tissue was stained by the immunoperoxidase technique with antibodies to IFN- $\gamma(A$ and $B)$, IL-4 ( $C$ and $D)$, and TNF- $\alpha(E$ and $F)$. Intense staining for IFN- $\gamma$ $(A)$ and IL-4 $(C)$ was observed in B10.M (DR3-) but not B10.M (DR3+) mice $(B$ and $D$ ). TNF- $\alpha$ immunoreactivity was similar in both groups at this time point ( $E$ and $F$ ). reduces demyelination via a mechanism unrelated to viral persistence.

Three distinct observations were made between the DR3+ transgenic mice and their littermate controls which may account for the differences observed in the extent of pathology between the groups: (a) DR3 + transgenic mice expressed the transgene on both infiltrating cells and CNS resident cells; $(b)$ at day 21 after infection, large amounts of IL-4 and IFN- $\gamma$ were observed in the lesions of nontransgenic but not in DR3+ transgenic mice; and (c) peptides derived from the DR3 gene are recognized as immunogenic in B10.M but not B10.M $(\mathrm{DR} 3+)$ mice. We have considered three possible mechanisms by which the severity of any immune-mediated disease can be modulated via a particular MHC class II gene. Expression of a specific class II allele may (a) result in the presentation of a peptide which could either be pathogenic or protective to the host; $(b)$ alter the cytokine response so that there is a change in the type or amount of a mediator(s) involved in either increas- ing or decreasing disease severity; or $(c)$ result in the deletion of a particular subset of $\mathrm{T}$ cells during thymic development. These proposed mechanisms are not mutually exclusive, and in this study may function together to reduce disease severity in the DR3 + mice.

In this model of TMEV-induced demyelination, the expression of the human DR3 transgene during development could have had profound effects on the T cell repertoire. Previous studies of B10.Q mice expressing this particular DR3 transgene demonstrated that some subsets of CD4+ $\mathrm{T}$ cells underwent positive selection (i.e., V $\beta 6$ and $\mathrm{V} \beta 8.1 / 8.2$ ), and

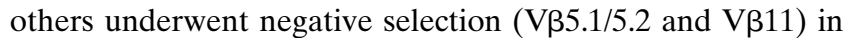
the presence of the transgene. Likewise, in the CD8+ $\mathrm{T}$ cell population, the $\mathrm{V} \beta 5.1 / 5.2+, \mathrm{V} \beta 8.1 / 8.2+$, and $\mathrm{V} \beta 11$ subpopulations were similarly skewed compared with nontransgenic littermate controls (32). The recognition of DR3 as self as well as the peptides derived from the DR3 molecule may have resulted in the deletion of $\mathrm{T}$ cells involved in some aspect of vi- 


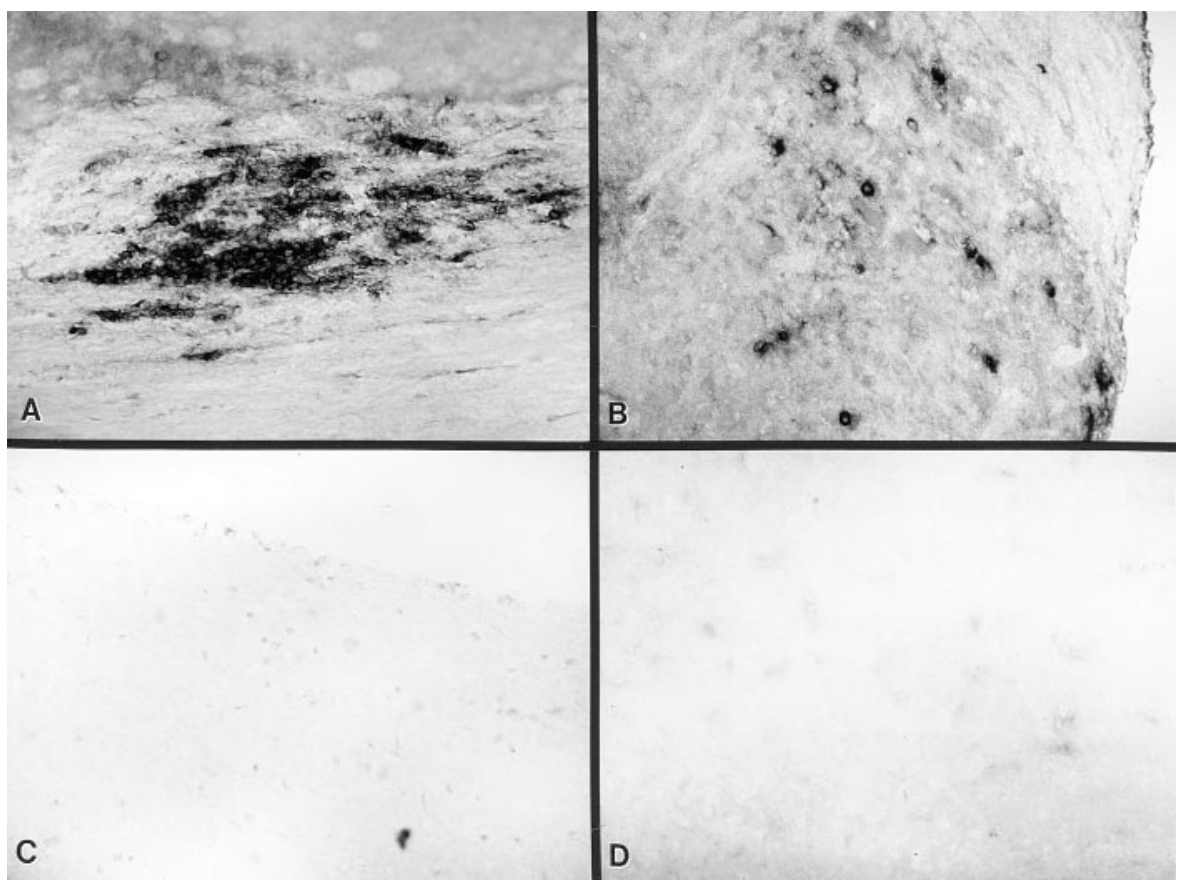

Figure 4. Expression of human HLA-DR3 transgene in the spinal cord. $(A)$ Infiltrating inflammatory cells in a lesion of an infected DR3 transgenic mouse (day 45) positive by immunoperoxidase staining for DR3 antigen. (B) No DR3-positive cells in a nontransgenic infected mouse. $(C)$ CNS resident cells, including neurons and astrocytes expressing HLA-DR3 at day 45 after infection. $(D)$ No staining in an uninfected B10.M (DR3+) mouse. rus-induced pathology. This possibility is supported by the demonstration that deletion of $\mathrm{V} \beta$ families influences the extent of demyelination after TMEV infection $(40,41)$. The results of the $\mathrm{T}$ cell proliferative assay (Table $\mathrm{V}$ ) demonstrate that mice on a B10.M background (susceptible to TMEVinduced demyelination) present and recognize peptide derived from the DR3 molecule. Furthermore, the B10.M (DR3+) mice do not mount a proliferative response to a DR3-derived peptide, demonstrating that a $\mathrm{T}$ cell population with the particular reactivity is deleted in these mice. Based on these in vitro responses, it is reasonable to conclude that the expression of DR3 in the transgenic mice would negatively select an autoreactive $T$ cell population in the HLA-DR3+ mice.

Alternatively, during the course of infection, epitopes may be generated that interact with DR3 to activate a subset of T cells not normally responding during TMEV infection. Studies of autoimmune thyroiditis have demonstrated that the HLADR3 molecule in these mice is capable of presenting peptide antigen (42). During TMEV infection, the peptides involved in this response may be derived from either virus or self. Activation of these peptide-specific T cells could potentially alter the balance of cytokines produced in the lesion, which may influence disease severity. As DR3+ and DR3- mice have an equal incidence of demyelinating disease after virus infection, we assume that those peptides critical for reducing disease severity are not involved in the onset of disease. The data demonstrating the delayed expression of IL-4 and IFN- $\gamma$ in the lesions of DR3 + mice indicate that the critical events for determining the differences in extent of demyelination occur between days 7 and 21 after TMEV infection. The extensive staining for IL- 4 and IFN- $\gamma$ observed in the lesions of DR3but not DR3 + mice supports a mechanism for the role of these cytokines in the pathology observed after TMEV infection. The presence of both $\mathrm{TH} 1$ and $\mathrm{TH} 2$ mediators in the lesions of
DR3 - mice suggests that the immunopathological mechanisms in this disease are the result of complex interactions between both $\mathrm{TH} 1$ and $\mathrm{TH} 2$ responses, as opposed to the absolute responses observed in some parasite or bacterial infections (43-45). These results may also explain, in part, previous observations which showed that the administration of exogenous cytokines which enhance either cellular or antibody responses (TNF- $\alpha$ [46] and IL-6 [47], respectively) ameliorates the extent of disease. In addition, treatment with anti-IFN- $\gamma$ has been shown to result in more severe disease in susceptible mice (48). Because there was no difference in the infectious virus titers, virus antigen expression, or virus RNA levels between the DR3+ and DR3- mice, this indicates that the mechanism for the reduction of demyelination was not the result of presentation by DR3 of TMEV-specific peptides important for clearing infection. A more likely hypothesis is that the immune response to the peptides (viral or host-derived) mediating immunopathology was suppressed. Because the "pathogenic epitopes" in this disease or the proteins from which they are derived are unknown, a direct test of this mechanism is not possible. Studies from another laboratory have identified virus-derived T cell epitopes in mice susceptible to TMEV (49, 50), but it is not known whether these epitopes are important in the strain of virus used in the experiments reported here, or whether they are critical for myelin destruction.

The observation that the DR3 transgene is expressed at high levels in resident CNS cells and in cells infiltrating the lesions supports a mechanism that includes direct involvement of the DR3 molecule in modulating pathology in the CNS. We propose that the mechanism involves altering the phenotype of the cells infiltrating the lesions, which results in the timing of when particular cytokines are localized to the lesions.

These data are the first to demonstrate that a human class II transgene decreases the pathology in a murine model of MS. 
By further examining the mechanism that results in reduced demyelination, it may be possible to gain insights into ways to prevent the progression of human MS.

\section{Acknowledgments}

The technical assistance of Julie Hanson and Michelle Smart in the breeding and typing of transgenic mice is greatly appreciated.

This work is supported by grants from the National Institutes of Health (NS-244180, NS-32129, and AI-14764) and grant RG 2203-B-6 from the National Multiple Sclerosis Society. K.M. Drescher is a fellow of the National Multiple Sclerosis Society. We also thank Mr. and Mrs. Eugene Applebaum and Ms. Kathryn Peterson for their generous financial support.

\section{References}

1. Black, C., D. Briggs, and K. Welsh. 1992. The immunogenetic background of scleroderma-an overview. Clin. Exp. Dermatol. 17:73-78.

2. Pryhuber, K.G., K.J. Murray, P. Donnelly, M.H. Passo, W.P. Maksymowych, D.N. Glass, E.H. Giannini, and R.A. Colbert. 1996. Polymorphism in the LMP2 gene influences disease susceptibility and severity in HLA-B27 associated juvenile rheumatoid arthritis. J. Rheumatol. 23:747-752.

3. Zanelli, E., C.J. Krco, J.M. Baisch, S. Cheng, and C.S. David. 1996. Immune response of HLA-DQ8 transgenic mice to peptides from the third hypervariable region of HLA-DRB1 correlates with predisposition to rheumatoid arthritis. Proc. Natl. Acad. Sci. USA. 93:1814-1819.

4. Zanelli, E., M.A. Gonzalez-Gay, and C.S. David. 1995. Could HLADRB1 be the protective locus in rheumatoid arthritis? Immunol. Today. 16: 274-278.

5. Fuks, A., S.J. Ono, E. Colle, and R.D. Guttmann. 1990. A single dose of the MHC-linked susceptibility determinant associated with the RT1u haplotype is permissive for insulin-dependent diabetes mellitus in the BB rat. Exp. Clin. Immunogenet. 7:162-169.

6. Stenszky, V., L. Kozma, C. Balazs, J.C. Bear, and N.R. Farid. 1986. The role of HLA antigens in the manifestation and course of Graves' disease. Mol. Biol. Med. 3:53-62.

7. Mustonen, J., J. Partanen, M. Kanerva, K. Pietila, O. Vapalahti, A. Pasternack, and N. Vaheri. 1996. Genetic susceptibility to severe course of nephropathia epidemica caused by Puumala hantavirus. Kid. Int. 49:217-221.

8. Simmons, A. H-2-linked genes influence the severity of herpes simplex virus infection of the peripheral nervous system. J. Exp. Med. 169:1503-1507.

9. Wolfgram, L.J., K.W. Beisel, A. Herskowitz, and N.R. Rose. 1986. Variations in the susceptibility of Coxsackievirus B3-induced myocarditis among different strains of mice. J. Immunol. 136:1846-1852.

10. Jersild, C., A. Svejgaard, and T. Fog. 1972. HLA antigens and multiple sclerosis. Lancet. i:1240-1242.

11. Ebers, G.C., K. Kukay, D.E. Bulman, A.D. Sadovnick, G. Rice, C. Anderson, H. Armstrong, K. Cousin, R.B. Bell, W. Hader, et al. 1996. A full genome screen in multiple sclerosis. Nat. Genet. 13:472-476.

12. Multiple Sclerosis Study Group. 1996. A complete genomic screen for multiple sclerosis underscores a role for the major histocompatibility complex. Nat. Genet. 13:469-471.

13. Sawcer, S., H.B. Jones, R. Feakes, J. Gray, N. Smaldon, J. Chataway, N. Robertson, D. Clayton, P.N. Goodfellow, and A. Compston. 1996. A genome screen in multiple sclerosis reveals susceptibility loci on chromosome $6 \mathrm{p} 21$ and 17q22. Nat. Genet. 13:464-468.

14. Ebers, G.C., and A.D. Sadovnick. 1994. Association studies in multiple sclerosis. J. Neuroimmunol. 53:117-122.

15. Duquette, P., F. Decary, J. Pleines, D. Boivin, G. Lamoureux, J.B. Cosgrove, and Y. Lapierre. 1985. Clinical sub-groups of multiple sclerosis in relation to HLA: DR alleles as possible markers of disease progression. Can. J. Neurol. Sci. 12:106-110.

16. Engell, T., N.E. Raun, M. Thomsen, and P. Platz. 1982. HLA and heterogeneity of multiple sclerosis. Neurology. 32:1043-1046.

17. Poser, S., G. Ritter, H.J. Bauer, H. Grosse-Wilde, E.K. Kuwert, and N.E. Raun. 1981. HLA antigens and the prognosis of multiple sclerosis. J. Neurol. 225:219-221.

18. British and Dutch Multiple Sclerosis Azathioprine Trial Group. 1988. Histocompatibility antigens in multiple sclerosis patients participating in a multicentre trial of azathioprine. J. Neurol. Neurosurg. Psychiatry. 51:412-415.

19. Runmarker, B., T. Martinsson, J. Wahlstrom, and O. Andersen. 1994. HLA and prognosis in multiple sclerosis. J. Immunol. 241:385-390.

20. Lipton, H.L. 1975. Theiler's virus infection in mice: an unusual biphasic disease leading to demyelination. Infect. Immun. 11:1147-1155.

21. Rodriguez, M., J.L. Leibowitz, and C.S. David. 1986. Susceptibility to Theiler's virus-induced demyelination. Mapping of the gene within the H-2D region. J. Exp. Med. 163:620-631.

22. Clatch, R.J., R.W. Melvold, S.D. Miller, and H.L. Lipton. 1985 Theiler's murine encephalomyelitis virus (TMEV)-induced demyelinating disease is influenced by the $\mathrm{H}-2 \mathrm{D}$ region: correlation with TMEV-specific delayed-type hypersensitivity. J. Immunol. 135:1408-1414.

23. Rodriguez, M., and C.S. David. 1985. Demyelination induced by Theiler's virus: influence of the H-2 haplotype. J. Immunol. 135:2145-2148.

24. Melvold, R.W., D.M. Jokinen, S.D. Miller, M.C. Dal Canto, and H.L. Lipton. 1987. H-2 genes in TMEV-induced demyelination, a model for multiple sclerosis. In Major Histocompatibility Genes and Their Role in Immune Function. C. David, editor. Plenum Publishing Corp., New York. 735-745.

25. Patick, A.K., L.R. Pease, C.S. David, and M. Rodriguez. 1990. Major histocompatibility complex-conferred resistance to Theiler's virus-induced demyelinating disease is inherited as a dominant trait in B10 congenic mice. $\mathrm{J} . \mathrm{Vi}$ rol. $64: 5570-5576$

26. Rodriguez, M., C.S. David, and L.R. Pease. 1987. The contribution of MHC gene products to demyelination by Theiler's virus. In Major Histocompatibility Genes and Their Role in Immune Function. C. David, editor. Plenum Publishing Corp., New York. 747-756.

27. Rodriguez, M., W.P. Lafuse, J.L. Leibowitz, and C.S. David. 1986. Partial suppression of Theiler's virus-induced demyelination in vivo by administration of monoclonal antibodies to immune-response gene products (Ia antigens). Neurology. 36:964-970.

28. Rodriguez, M., and S. Sriram. 1988. Successful therapy of TMEV-induced demyelination (DA strain) with monoclonal anti-lyt 2.2 antibody. J. Immunol. 140:2950-2955.

29. Welsh, C.J., P. Tonks, A.A. Nash, and W.F. Blakemore. 1987. The effect of L3T4 T cell depletion on the pathogenesis of Theiler's murine encephalomyelitis virus infection in CBA mice. J. Gen. Virol. 68:1659-1667.

30. Njenga, M.K., K.D. Pavelko, J. Baisch, X. Lin, C.S. David, J.L. Leibowitz, and M. Rodriguez. 1996. Theiler's virus persistence and demyelination in major histocompatibility complex class II-deficient mice. J. Virol. 70:1729-1737.

31. Rodriguez, M., M.L. Pierce, and E.A. Howie. 1987. Immune response gene products (Ia antigens) on glial and endothelial cells in virus-induced demyelination. J. Immunol. 138:3438-3442.

32. Strauss, G., D.A.A. Vignali, G. Schonrich, and G.L. Hammerling. 1994 Negative and positive selection by HLA-DR3(DRw17) molecules in transgenic mice. Immunogenetics. 40:104-108.

33. Pierce, M., and M. Rodriguez. 1989. Erichrome stain for myelin on osmicated tissue embedded in glycol methacrylate plastic. J. Histotechnol. 12:35-36.

34. Rodriguez, M., J.L. Leibowitz, H.C. Powell, and P.W. Lampert. 1984. Neonatal infection with the DA strain of Theiler's murine encephalomyelitis virus (TMEV). Lab. Invest. 49:672-679.

35. Rodriguez, M., A.J. Dunkel, R.L. Thiemann, J.L. Leibowitz, M. Zijlstra, and R. Jaenisch. 1993. Abrogation of resistance to Theiler's virus-induced demyelination in $\mathrm{H}-2^{\mathrm{b}}$ mice deficient in $\beta 2$-microglobulin. J. Immunol. 151:266276.

36. Miller, D.J., M.K. Njenga, P.D. Murray, J.L. Leibowitz, and M. Rodriguez. 1996. A monoclonal natural autoantibody that promotes remyelination suppresses central nervous system inflammation and increases virus expression after Theiler's virus-induced demyelination. Int. Immunol. 8:131-141.

37. Krco, C.J., T.G. Beito, and C.S. David. 1992. Determination of tolerance to self $\mathrm{E}$ alpha peptides by clonal elimination of $\mathrm{H}-2 \mathrm{E}$ reactive $\mathrm{T}$ cells and antigen presentation by H-2A molecules. Transplantation (Baltimore). 54:920-923.

38. Lindsley, M., and M. Rodriguez. 1989. Characterization of the inflammatory response in the CNS of mice susceptible or resistant to demyelination by Theiler's virus. J. Immunol. 142:2677-2682.

39. Rodriguez, M., J.J. Kenny, R.L. Thiemann, and G.E. Woloschak. 1990. Theiler's virus-induced demyelination in mice immunosuppressed with antiIgM and in mice expressing the xid gene. Microb. Pathg. 8:23-35.

40. Rodriguez, M., G.H. Nabozny, R.L. Thiemann, and C.S. David. 1994 Influence of deletion of $\mathrm{T}$ cell receptor $\mathrm{V} \beta$ genes on Theiler's virus model of multiple sclerosis. Autoimmunity. 19:221-230.

41. Rodriguez, M., A.K. Patick, L.R. Pease, and C.S. David. 1992. Role of T cell receptor $\mathrm{V} \beta$ genes in Theiler's virus-induced demyelination of mice. J. Immunol. 148:921-927.

42. Kong, Y.C.M., L.C. Lomo, R.W. Motte, A.A. Giraldo, J. Baisch, G. Strauss, G.J. Hammerling, and C.S. David. 1996. HLA-DRB1 polymorphism determines susceptibility to autoimmune thyroiditis in transgenic mice: definitive association with HLA-DRB1*0301 (DR3) gene. J. Exp. Med. 184:11671172.

43. Locksley, R.M., and J.A. Louis. 1992. Immunology of leishmaniasis. Curr. Opin. Immunol. 4:413-418.

44. Sher, A., R.T. Gazzinelli, I.P. Oswald, M. Clerici, M. Kullberg, E.J. Pearce, J.A. Berzofsky, T.R. Mosmann, S.L. James, and H.C. Morse III. 1992. Role of T-cell derived cytokines in the downregulation of immune responses in parasitic and retroviral infection. Immunol. Rev. 127:183-204.

45. Munk, M.E., and M. Emoto. 1995. Functions of T-cell subsets and cytokines in mycobacterial infections. Eur. Resp. J. Suppl. 20:668S-675S.

46. Paya, C.V., P.J. Leibson, A.K. Patick, and M. Rodriguez. 1990. Inhibition of Theiler's virus-induced demyelination in vivo by tumor necrosis factor alpha. Int. Immunol. 2:909-913. 
47. Rodriguez, M., K.D. Pavelko, C.W. McKinney, and J.L. Leibowitz. 1994. Recombinant human IL-6 suppresses demyelination in a viral model of multiple sclerosis. J. Immunol. 153:3811-3821.

48. Pullen, L.C., S.D. Miller, M.C. Dal Canto, P.H. Van der Meide, and B.S. Kim. 1994. Alteration in the level of interferon-gamma results in acceleration of Theiler's virus-induced demyelinating disease. J. Neuroimmunol. 55:143-152.

49. Gerety, S.J., M.K. Rundell, M.C. Dal Canto, and S.D. Miller. 1994. Class II-restricted T cell responses in Theiler's murine encephalomyelitis virus- induced demyelinating disease. V. Mapping of a dominant immunopathologic VP2 T cell epitope in susceptible SJL/J mice. J. Immunol. 152:908-918.

50. Gerety, S.J., M.K. Rundell, M.C. Dal Canto, and S.D. Miller. 1994 Class II-restricted T cell responses in Theiler's murine encephalomyelitis virusinduced demyelinating disease. VI. Potentiation of demyelination with and characterization of an immunopathologic CD4+ T cell line specific for an immunodominant VP2 epitope. J. Immunol. 152:919-929. 